\title{
Effects of Qing'E Formula on the Expression of Bone Metabolic Markers and VDR mRNA in Postmenopausal Osteoporosis Patients
}

\author{
Bo Shuai, Yanping Yang*, Lin Shen", Hui Ke \\ Department of Integrated Traditional Chinese and Western Medicine, Union Hospital, Tongji Medical College, \\ Huazhong University of Science and Technology, Wuhan, China \\ Email: " bobo3137@126.com
}

Received 20 May 2014; revised 28 June 2014; accepted 13 July 2014

Copyright $@ 2014$ by authors and Scientific Research Publishing Inc.

This work is licensed under the Creative Commons Attribution International License (CC BY). http://creativecommons.org/licenses/by/4.0/

(c) (7) Open Access

\begin{abstract}
Objectives: To study the partial mechanism of treating postmenopausal osteoporosis patients (POPs) using the ancient recipe of Qing'E Formula (QEF) by observing its effects on bone metabolic markers and VDR mRNA expression in primary POPs. Methods: Analysis was performed on 120 outpatient and inpatient POPs treated in our hospital between January and October 2013, where the patients were randomly divided into Qing'E group (QEF + Caltrate), Calcitriol group (Caltrate + Calcitriol soft capsules), and Compare group (Caltrate), each with a follow-up period of 1 year. Statistical analysis was then performed on bone mineral density, blood bone metabolic markers $(\beta$-CTX, N-MID, T-PINP) and changes in VDR mRNA expressions in the POPs before and after the treatments. Results: Prior to the treatments, bone mineral density and blood $\beta$-CTX, N-MID, T-PINP and VDR mRNA expression in the 3 groups of POPs exhibited no statistically significant differences, and the blood $\beta$-CTX, N-MID, T-PINP and VDR mRNA expression in the control group showed no statistically significant differences before and after the treatments. There were no significant differences in bone mineral density in the Qing'E group and the Calcitriol group before and after the treatments whereas the bone mineral density decreased in the control group after the treatments. As for blood $\beta$-CTX, N-MID, T-PINP and VDR mRNA expression, the measurements in POPs in the Qing'E group and the Calcitriol group were significantly higher than that of the control group. Conclusion: By adjusting the VDR mRNA expression, the QEF, a kidney-invigorating Chinese herbal formula, is capable of activating bone metabolism to prohibit further losses of bone mass, thereby preventing the deterioration of osteoporosis.
\end{abstract}

\section{Keywords}

Qing'E Formula, VDR mRNA, Osteoporosis, Bone Metabolism, Bone Density

\footnotetext{
*This author contributed equally to this work.

${ }^{\#}$ Corresponding author.
}

How to cite this paper: Shuai, B., et al. (2014) Effects of Qing'E Formula on the Expression of Bone Metabolic Markers and VDR mRNA in Postmenopausal Osteoporosis Patients. Chinese Medicine, 5, 145-152. 


\section{Introduction}

With the aging of population in our society, osteoporosis and other metabolic bone diseases and their complications have become one of the key factors affecting the healthy living of the elderly population and increasing the society’s economic burdens. The QEF, a Chinese herbal formula, has the efficacy of "nourishing livers and kidneys", “increasing energy and blood" and "regenerating bone marrows". Preliminary studies by the research team showed that QEF could effectively raise $1,25(\mathrm{OH})_{2} \mathrm{D}_{3}$ and mRNA expression in the blood of ovariectomized rats, thereby effectively controlling osteoporosis. This study proposes the further study on the partial mechanism of treating POPs using ancient recipe QEF by studying its effects on the special sequence of $\beta$ collagen in blood markers of bone metabolism ( $\beta$-Crosslaps, $\beta$-CTX), $\mathrm{N}$-terminal osteocalcin (N-MID), total N-terminal propeptide of type I procollagen (T-PINP) and VDR mRNA expression in POPs.

\section{Materials and Methods}

\subsection{Research Objects and Grouping}

The research objects were 120 primary POPs, with an average age of $53 \pm 6$ years old. The subjects, comprising of either inpatient or outpatient patients of the hospital, were randomly segregated into three groups, i.e. the Qing'E group, who were given the treatment combination of Caltrate and QEF; the Calcitriol group, who were given the treatment combination of Caltrate and Calcitriol soft capsules; and the compare group, who were only given Caltrate treatments. The groups, consisting of 40 POPs each, were given treatments for the period of 1 year. The administering method of medicine is in the "Experiment Medicine" section. This study has been approved by the ethics committee of Wuhan Union Hospital Research Center, along with endorsement from all personnel participating in the study. The participating POPs had also refrained from taking any medicine that affects bone metabolism and coagulation functions two weeks prior to the determination.

\subsection{Experiment Medicine}

The making process of experiment medicine-ancient herbal formula QEF: The ingredients include the following: Eucommia (fried with salt) 480 g, Psoralen (fried with salt) 240 g, walnut (fried) 150 g, Salvia 240 g and garlic 120 g. Garlic was steamed, dried, crushed into fine powder with Eucommia, Salvia and Psoralen and then sifted. Walnut was then mashed and grinded with the above powder before the mixture was further sifted and mixed. For every 100 grams of the powder, 50 - 70 grams of refining honey was added for producing the Qing'E pill. The experiment objects were then allocated randomly and received treatments under the experiment medicine or the control medicine. QEF were administered three times daily with warm water whereas Calcitriol soft capsules were administered twice a day, with 1 pill for each intake. In addition to the experiment medicine, the experiment groups and the control group were also given 1 piece of Caltrate daily to ensure patients' compliance and facilitate better treatments. Any traditional Chinese medicine or modern western medicine that affects bone metabolism and coagulation functions were prohibited during the course of the observation period.

\subsection{Experiment Methods}

\subsubsection{Information Collection and Bone Density Testing}

Physical examination and survey results of all patients were recorded in detail before and after the treatments. The U.S. made Hologic 2000 Plus Dual-energy X-ray Bone Densitometer and conventional standard software were used to measure bone density at the lumbar spine, the proximal femur, Ward', and greater trochanter. Patients were placed in the position according to requirements, followed by analysis of the images and the results were reported with reference to the WHO diagnostic criteria $\left(\mathrm{g} / \mathrm{cm}^{2}\right)$. The results were automatically printed and exported from the equipment's supporting printer. The bone density of all patients was measured by one single person using the same equipment to minimize variations in statistical analysis.

\subsubsection{Measurement of Blood Bone Metabolic Markers in Patients}

Blood samples were drawn from the cubital vein in patients at approximately 10 a.m. every day. Part of the collected blood sample was then centrifuged for 15 minutes (speed: $1000 \times \mathrm{g}$, temperature $2^{\circ} \mathrm{C}-8^{\circ} \mathrm{C}$ ) and stored in refrigerator at $-70^{\circ} \mathrm{C}$ while bone metabolic markers $\beta$-CTX, N-MID, T-PINP in blood were collected, all the 
blood were delivered to the same central biochemical lab for tests following the collection, and the tests were conducted strictly according to the instructions listed on the test kits.

\subsubsection{Measurement of Blood Adiponectin mRNA in Patients}

VDR mRNA expression was measured in the other portion of the blood samples, the VDR primer: under the role of reverse transcriptase M-MLV, the m RNA reverse transcribed into c-DNA molecules. The reaction system was as follows: $\mathrm{H}_{2} \mathrm{O} 5.5 \mu \mathrm{l}$, Oligo (dT18) $1.0 \mu$ l, TRNA $6.0 \mu \mathrm{l}$, RNasin $0.5 \mu \mathrm{l}, 5 \times$ buffer $4.0 \mu \mathrm{l}, 10 \mathrm{mM}$ dNTP $2.0 \mu$ l, RTase $1.0 \mu \mathrm{l}$; the reaction conditions: 42 Celsius for 60 minutes, and 95 Celsius for 5 minutes. Quantitative PCR reaction system as follows: cDNA $1 \mu \mathrm{l}$, Buffer $10 \times 5 \mu \mathrm{l}, \mathrm{MgCl}_{2}(25 \mathrm{mM}) 7 \mu \mathrm{l}$, dNTP $10 \mathrm{mM}$

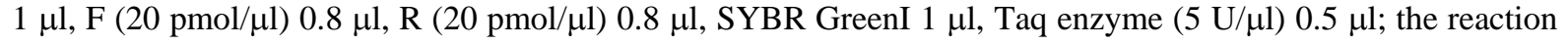
conditions: 94 Celsius for 30 minutes, 53 Celsius for 30 minutes, and 72 degree for 30 minutes for 50 cycles. Ct values were calculated and analyzed: the sample relative value $=2^{-(\Delta \mathrm{Ct} \beta \text {-actin- } \Delta \mathrm{Ct} \text { test sample })}, \Delta \mathrm{Ct}=\mathrm{Ct}$ negative control - Ct sample, $\Delta \Delta \mathrm{Ct}=\Delta \mathrm{Ct} \beta$-actin $-\Delta \mathrm{Ct}$ sample $=\mathrm{Ct}$ sample $-\mathrm{Ct} \beta$-actin .

\subsection{Statistical Methods}

Statistical Methods: Test results were represented by $\bar{x} \pm S$, statistical methods such as analysis of variance, paired sample $t$ test and t-test for independent samples were used for testing and analysis, $p<0.05$ represented statistical significance and data was analyzed using the SPSS13.0 software.

\section{Results}

\subsection{BMD Changes in the 3 Groups of Patients before and after Treatments}

There was no statistically significant difference in BMD among the three groups of patients before the treatments. After the treatments, the Qing'E group of patients saw slight increases in the BMD in lumbar spine, femoral neck, ward and the greater trochanter when compared with the measurements before the treatment; however, the difference was not statistically significant. There was no difference in the BMD in lumbar spine, femoral neck, ward and the greater trochanter in the Calcitriol group before and after the treatments whilst the BMD in these same parts saw significant decrease in the control group. There was no difference in BMD in lumbar spine, femoral neck, ward and the greater trochanter between the Qing'E group and the Calcitriol group, whilst, the measurements in the control group were significantly lower than the above 2 groups (Figure 1).

\subsection{Changes in Bone Metabolic Markers $\beta$-CTX, N-MID and T-PINP Expression in the 3 Groups of Patients before and after the Treatments}

The $\beta$-CTX, N-MID and T-PINP expression in the blood of the 3 groups of patients had no statistical significance before the treatments. After the treatments, there was no difference in the content of $\beta$-CTX, N-MID and T-PINP between the Qing'E and Calcitriol groups, however, the post-treatment contents were lower after treatments in both groups. There was no statistically significant difference in $\beta$-CTX, N-MID and T-PINP in the control group before and after the treatments, and the content in this group were significantly higher than that of the Qing’E and Calcitriol groups (Figure 2).

\subsection{Difference in VDR mRNA Expression in the 3 Groups of Patients before and after the Treatments}

There was no significant difference in VDR mRNA expression among the 3 groups of patients before the treatments. The post-treatment VDR mRNA expression in the Qing'E and Calcitriol groups were higher after treatments, but there was no significant difference between the 2 groups. There was no difference in VDR mRNA expression in the control group before and after treatments (Figures 3(a)-3(c)).

\section{Discussion}

Due to the withering of ovary, older women experience sharp decrease in estrogen in the body, which leads to significant negative balance of bone metabolism where the rate of bone resorption becomes faster than bone formation, resulting in osteoporosis. Postmenopausal women experience significant reduction in estrogen, which 


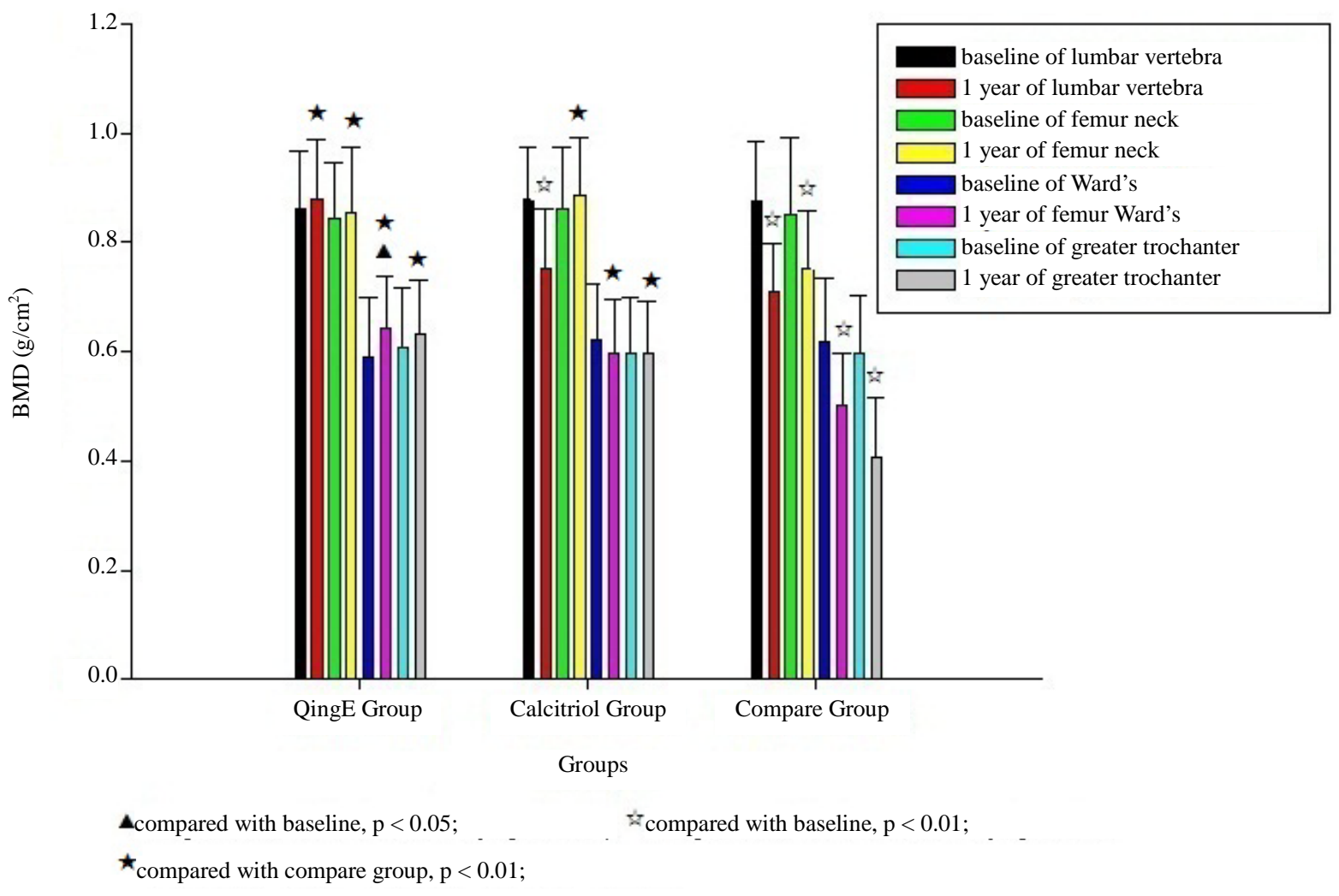

Figure 1. BMD changes in the 3 groups of patients before and after treatments.

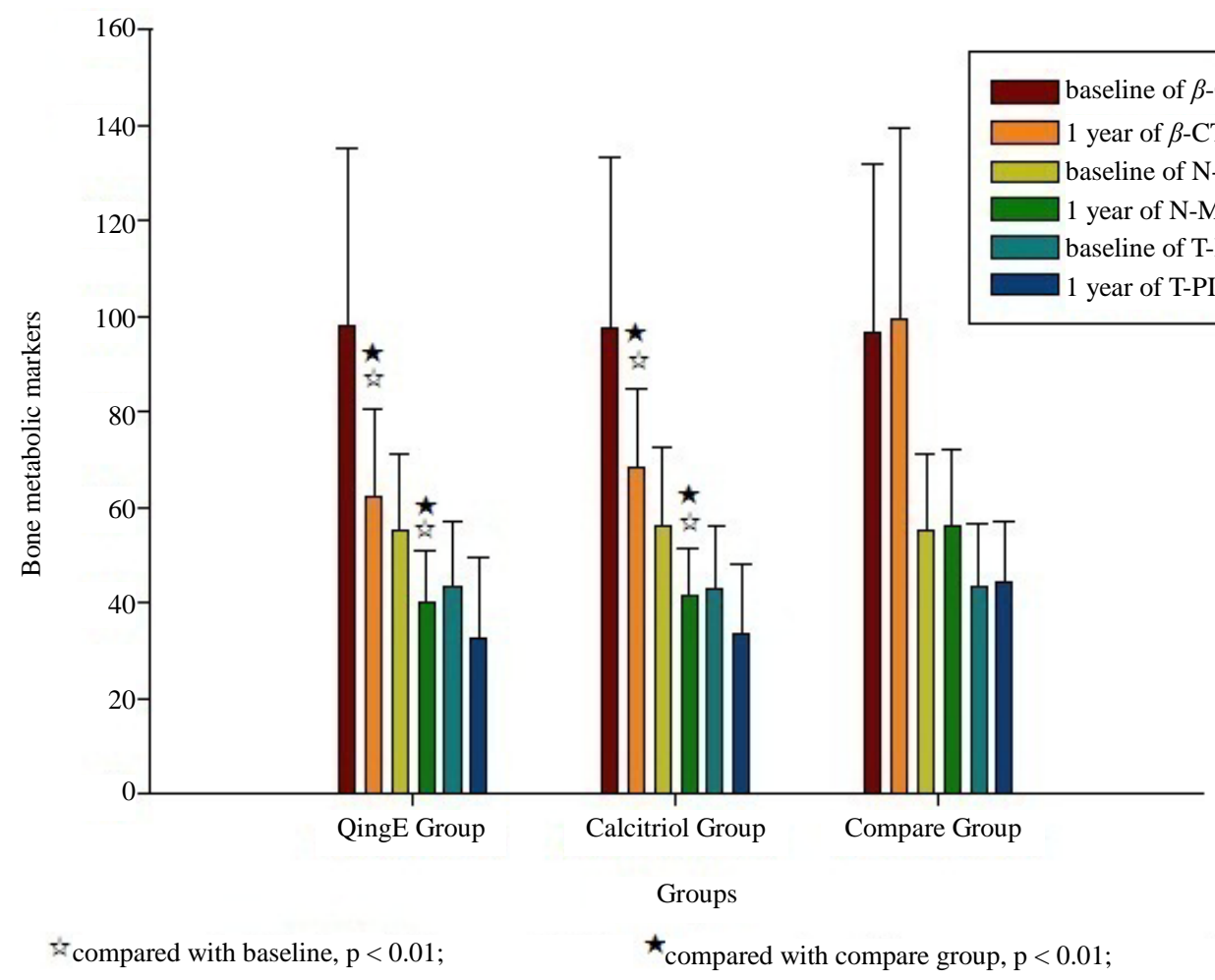

Figure 2. Changes in bone metabolic markers $\beta$-CTX, N-MID and T-PINP expression in the 3 groups of patients before and after the treatments. 

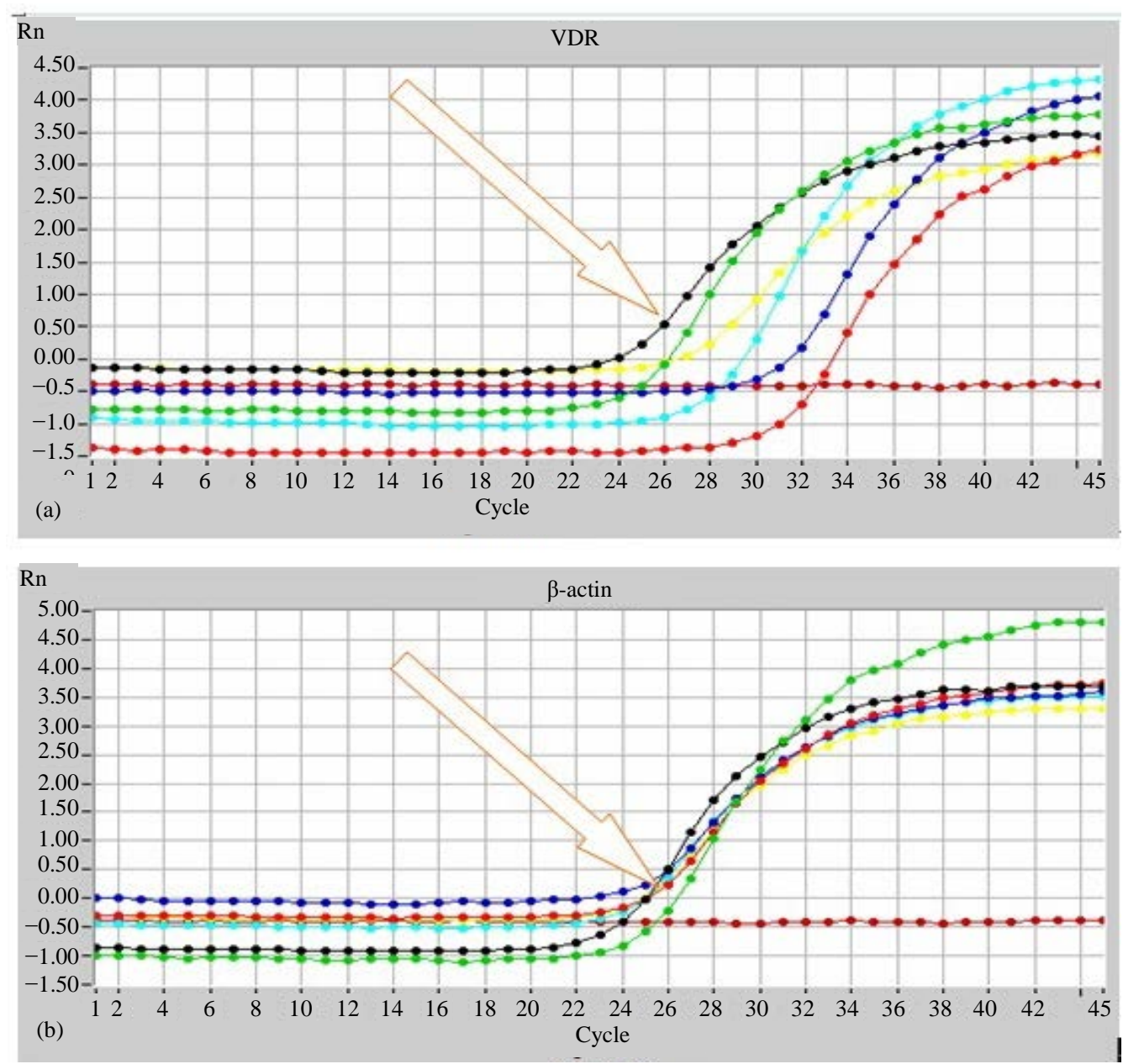

(a)

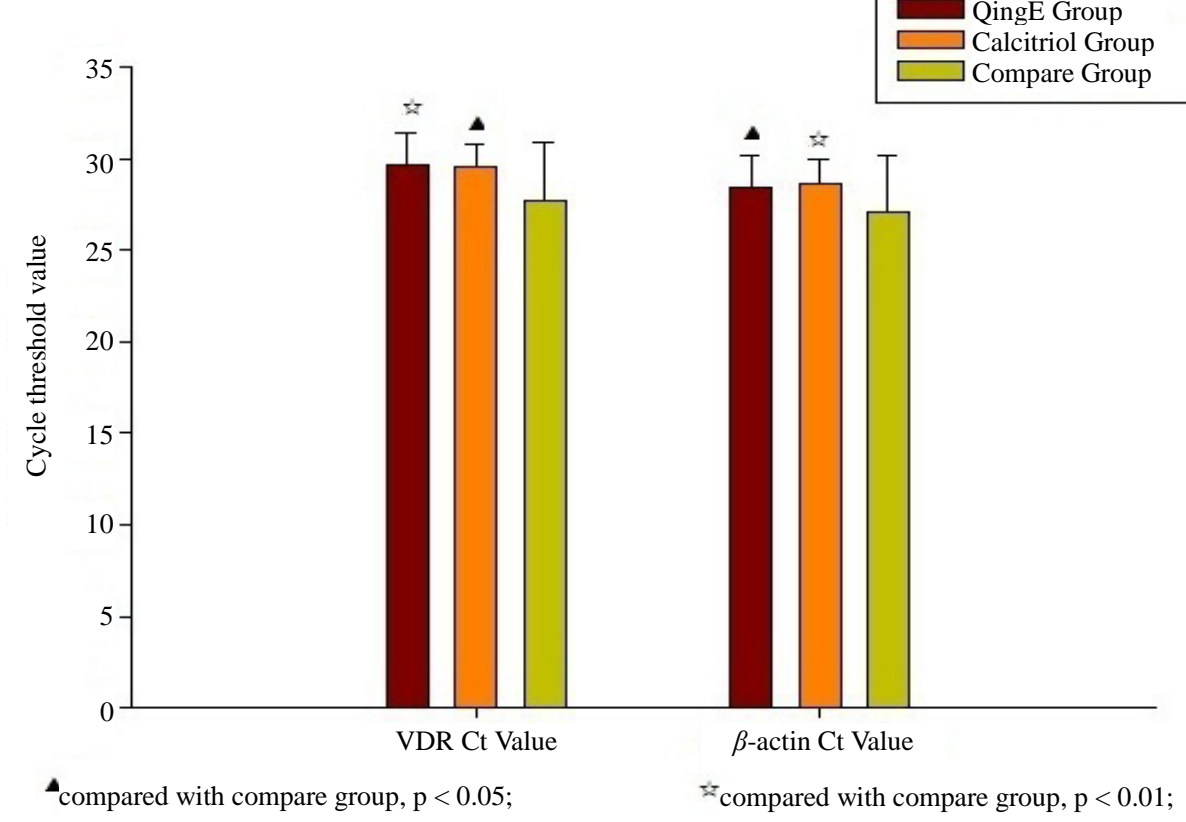

${ }^{\star}$ compared with compare group, $\mathrm{p}<0.05$;

QingE Group $\square$ Calcitriol Group

(b) 


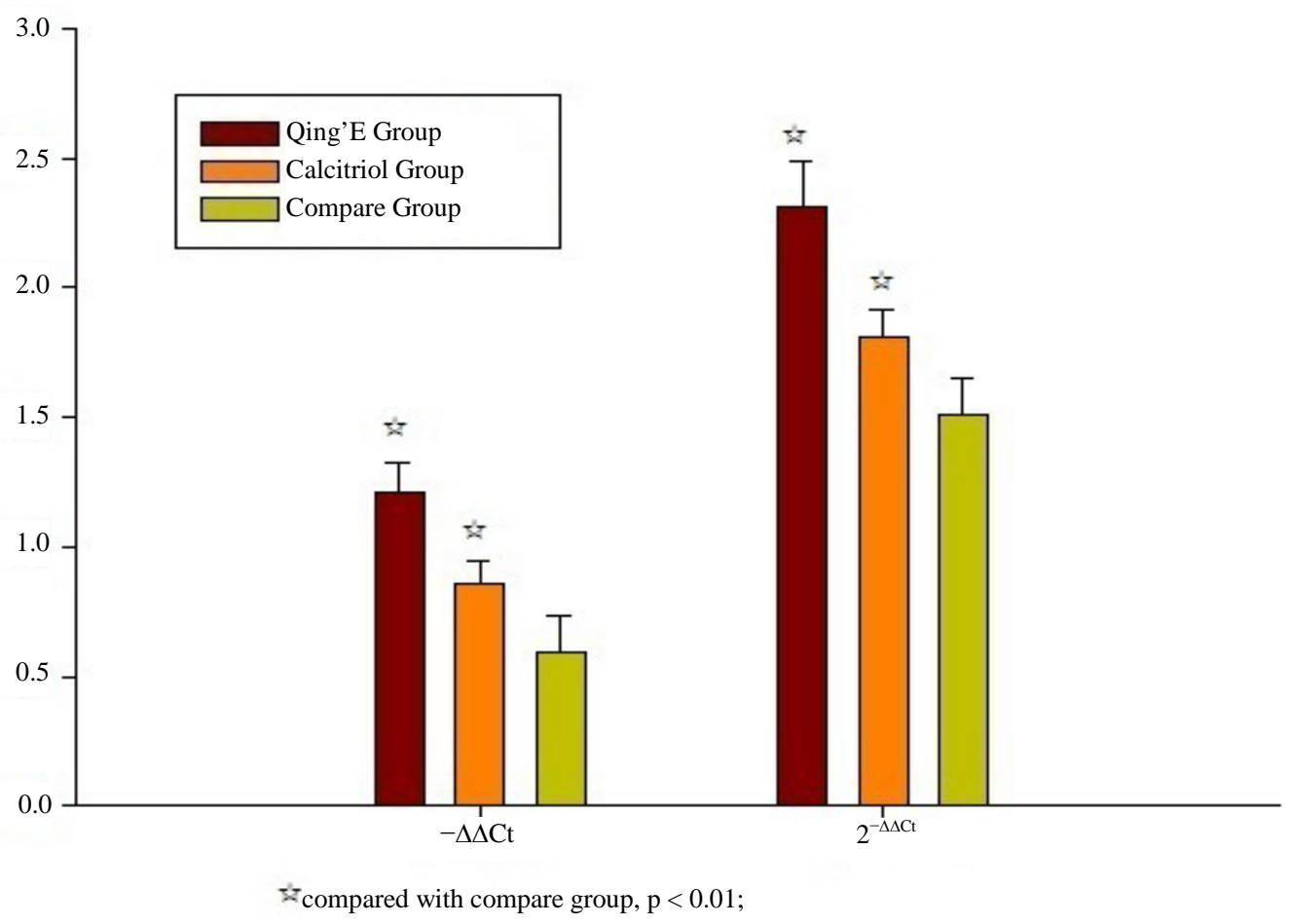

(c)

Figure 3. Difference in VDR mRNA expression in the 3 groups of patients before and after the treatments.

inhibits the secretion of parathyroid hormone (PTH) and the reduction of PTH creates barriers to the activation of $1 \alpha$-hydroxylase in livers, which would in turn lead to decrease in the synthesis of $1,25(\mathrm{OH})_{2} \mathrm{D}_{3}$. Addition of active vitamin $\mathrm{D}_{3}$ in the treatment for postmenopausal women could promote intestinal absorption of calcium and enhance the cellular activity of osteoclasts and osteoblasts, thereby promoting the mineralization of bones, bone formation and the absorption of the old bones. $1,25(\mathrm{OH})_{2} \mathrm{D}_{3}$ is the active form of the most bioactive vitamin D in the body. The biological activity of vitamin D is mainly the result of the interaction of $1,25(\mathrm{OH})$ and the VDR of the nucleus in target organs. The combination of $1,25(\mathrm{OH})_{2} \mathrm{D}_{3}$ and VDR forms a hormone-receptor complex, which would combine with the hormone response elements in the VDR gene to regulate VDR expression through the interaction with other regulatory factors [1] [2]. Researches have shown that vitamin D receptor gene polymorphism is closely associated with bone density of Han race in Guangxi, Beijing, Wuhan, Fujian and Guangzhou and other racial groups [3]-[5]. Preliminary studies by the research team showed that QEF could effectively suppress VDR mRNA expression in the kidney of ovariectomized rats to promote the expression of vitamin D and the absorption of calcium, thereby effectively controlling postmenopausal osteoporosis [6] [7]. Research has shown that the relative expression of VDR mRNA in the kidney of ovariectomized rats is significantly lower than the normal control group, which is consistent with the findings of this study [8]. VDR mRNA expression in the figure has significantly strengthened in the blood of active vitamin $\mathrm{D}_{3}$ treatment group (Calcitriol group). This might be attributable to the direct supplement of active vitamin $\mathrm{D}_{3}$, which has led to the significant increase in the content of "exogenous" $1,25(\mathrm{OH})_{2} \mathrm{D}_{3}$ in the blood, which in turn leads to the increase in VDR mRNA expression. There is no significant difference in VDR mRNA between QEF and Calcitriol groups, suggesting the possibility of including "kidney essence”, etc. in QEF to increase VDR mRNA expression, thereby achieving the same effects as Calcitriol soft capsules.

In "Qing'E Pill” prescription, Eucommia ulmoides oliv is warm-natured, with the effect of making kidney and bones strong, so in the book "Ben Cao Ji Yan", there was such a description, "Eucommia ulmoides oliv is only herb to strengthen lower energizer, remove pains of foots and legs, and fix knees and loins.” Also in another ancient book named "Ben Cao Jing Shu”, it was said "Psoralea corylifolia can warm the kidney, raise 'Yang' from 'Yin', raise ‘fire' from ‘soil', can cure kidney weakness and difficulties in sitting up.” In “Ju Fang Qing’E Pill”, 
it described that "Juglans regia can invigorate the kidney and promote blood circulation, garlic bulb can make the retention pass and dredge collaterals... The combination of these four kinds of herb is called 'Qing' $E$ Pill', which can not only cure kidney weakness and difficulties in sitting up, but also strengthen bone and musculature, promote blood circulation, make hair dark, and bring a good look.” After thousands years of clinical validation, Qing'E Pill was effective, safe and economical, however, there was a lack of specific mechanism research.

The $\beta$-CTX, N-MID and T-PINP are three types of bone metabolic markers recommended by the International Osteoporosis Foundation (IOF). The $\beta$-CTX is the organic component of bone matrix, of which $90 \%$ are type I collagen synthesized in bones, but are also decomposed into degradation products that are released into the blood. The $\beta$-CTX is a typical product of collagen degradation of type I collagen. The examination of $\beta$-CTX can be used for monitoring the anti-resorptive treatment for osteoporosis or other bone diseases, and the efficacy can be reflected within several weeks. The N-MID are produced by osteoblasts during bone synthesis. Both complete osteocalcin and large N-MID fragments are present in the blood; however, the former is unstable and may split and degrade into the latter. The N-MID osteocalcin is regarded as a marker of bone synthesis, which can be used in conjunction with $\beta$-CTX for monitoring the treatment of diseases such as osteoporosis. The determination of the concentration of total PINP in the blood is one of the test items of bone markers, which can be used for monitoring the treatment of osteoporosis in postmenopausal women clinically.

The biological activity of vitamin D is mainly the result of the interaction of $1,25(\mathrm{OH})$ and the VDR of the nucleus in target organs. Vitamin D can enhance osteoblast activity in bone metabolism and promote the formation of bone mineralization. In addition, it also has positive effects on relatively mature osteoblasts, such as increasing the expression of OC and ALP, promoting the mRNA expression of type I collagen of osteoblast in the late maturity stage and the secretion of type I collagen, promoting the synthesis and secretion of osteoblasts and the mineralization of bone matrix, as well as improving the quality and quantity of bones.

The results of this study show that QEF can reduce bone resorption through the adjustment of VDR mRNA expression. This experiment uses stable and effective active vitamin $\mathrm{D}_{3}$ reported in literatures as the reference drug for the control study [9]-[11]. Data shows similar drug efficacy between QEF and active vitamin $\mathrm{D}_{3}$, as both are able to increase VDR mRNA expression in the body in reducing bone resorption and strengthening the quality and quantity of bones. The results suggest that the ancient recipe QEF has comparable efficacy in the prevention and treatment of postmenopausal osteoporosis as appropriate supplement of active vitamin $\mathrm{D}_{3}$.

\section{Acknowledgements}

The authors would like to thank LV Lin for his expertise and assistance in performing the blood collection, and Wang Quanshen for his technical assistance.

\section{Research Support}

Innovation Fund of Huazhong University of Science and Technology (Item Number: 2013QN235); National Natural Science Foundation of China (Project Number: 81273907 and 81072493).

\section{References}

[1] Fan, L.Y., Tu, X.Q., Zhu, Y., Zhou, L., Pfeiffer, T., Feltens, R., Stoecker, W. and Zhong, R. (2005) Genetic Association of Vitamin D Receptor Polymorphisms with Autoimmune Hepatitis and Primary Biliary Cirrhosis in the Chinese. Journal of Gastroenterology and Hepatology, 20, 249-255. http://dx.doi.org/10.1111/j.1440-1746.2005.03532.x

[2] Van Etten, E. and Mathieu, C. (2005) Immunoregulation by 1,25-Dihydroxyvitamin $\mathrm{D}_{3}$ : Basic Concepts. The Journal of Steroid Biochemistry and Molecular Biology, 97, 93-101. http://dx.doi.org/10.1016/j.jsbmb.2005.06.002

[3] Zhou, Y., Zhang H. and Cai D.H. (2005) Influence of Estrogen and Progesteron on Bone Metabolism and Renal Expression of 1,25-Dihydroxyvitamin $\mathrm{D}_{3}$ Receptors mRNA in Ovariectomized Rats. Academic Journal of Second Military Medical University, 26, 1270-1273.

[4] Municio, M.J. and Traba, M.L. (2004) Effects of 24, 25-(OH) $)_{2} \mathrm{D}_{3}, 1,25-(\mathrm{OH})_{2} \mathrm{D}_{3}$ and 25(OH) $\mathrm{D}_{3}$ on Alkaline and Tartrate-Resistant Acid Phosphatase Activities in Fetal Rat Calvaria. Journal of Physiology and Biochemistry, 60, $219-224$. http://dx.doi.org/10.1007/BF03167032

[5] Uchida, M., Shima, M., Chikazu, D., Fujieda, A., Obara, K., Suzuki, H., Nagai, Y., Yamato, H. and Kawaguchi, H. (2001) Transcriptional Induction of Matrix Metalloproteinase-13 (Collagenase-3) by 1 Alpha,25-Dihydroxyvitamin $\mathrm{D}_{3}$ in Mouse Osteoblastic MC3T3-E1 Cells. Journal of Bone and Mineral Research, 16, 221-230. 
http://dx.doi.org/10.1359/jbmr.2001.16.2.221

[6] Shuai, B., Shen, L., Yang, Y.P., Xie, J., Zhou P.Q., Xu X.J., Li, C.G. and Wu, M.X. (2012) Effect of Bushenhuoxue Decoction on the Osteogenic Differentiation of Bone Marrow Stromal Cells in the Steroid Induced Osteonecrosis of the Femoral Head. Research of Integrated Traditional Chinese and Western Medicine, 4, 297-301.

[7] Shuai, B., Shen, L., Yang, Y.P., Xie, J., Shou, Z.X. and Wei, B. (2010) Low Plasma Adiponectin as a Potential Biomarker for Osteonecrosis of the Femoral Head. The Journal of Rheumatology, 37, 2151-2155. http://dx.doi.org/10.3899/jrheum.100342

[8] Guo, L.J., Luo, X.H., Xie, H., Zhou, H.D. and Liao, E.Y. (2005) The Effects of 1Alpha,25-Dihydroxyvitamin $D_{3}$ in Regulation Matrix Metalloproteinase and Tissue Metalloproteinase Inhibitors in Human Osteoblasts. Zhonghua Nei Ke Za Zhi, 44, 125-128.

[9] Xue, Z.W., Shang, X.M., Lv, S.H., Xu, H., Zhang, Q. and Wang, C. (2013) Effects of Shenshao Decoction on the Inflammatory Response in The aorta of a Rat Atherosclerotic Model. Chinese Journal of Integrative Medicine, 19, 347352. http://dx.doi.org/10.1007/s11655-013-1457-z

[10] Yao, F.A., Dobs, A.S. and Brown, T.T. (2006) Alternative Therapies for Osteoporosis. The American Journal of Chinese Medicine, 34, 721. http://dx.doi.org/10.1142/S0192415X06004235

[11] Ling, J.Y., Shen, L., Liu, Q., Xue, S., Ma, W., Wu, H., Li, Z.X. and Zhu, R. (2013) Changes in Platelet GPIba and ADAM17 during the Acute Stage of Atherosclerotic Ischemic Stroke among Chinese. Journal of Huazhong University of Science and Technology (Medical Sciences), 33, 438-442. 
Scientific Research Publishing (SCIRP) is one of the largest Open Access journal publishers. It is currently publishing more than 200 open access, online, peer-reviewed journals covering a wide range of academic disciplines. SCIRP serves the worldwide academic communities and contributes to the progress and application of science with its publication.

Other selected journals from SCIRP are listed as below. Submit your manuscript to us via either submit@scirp.org or Online Submission Portal.
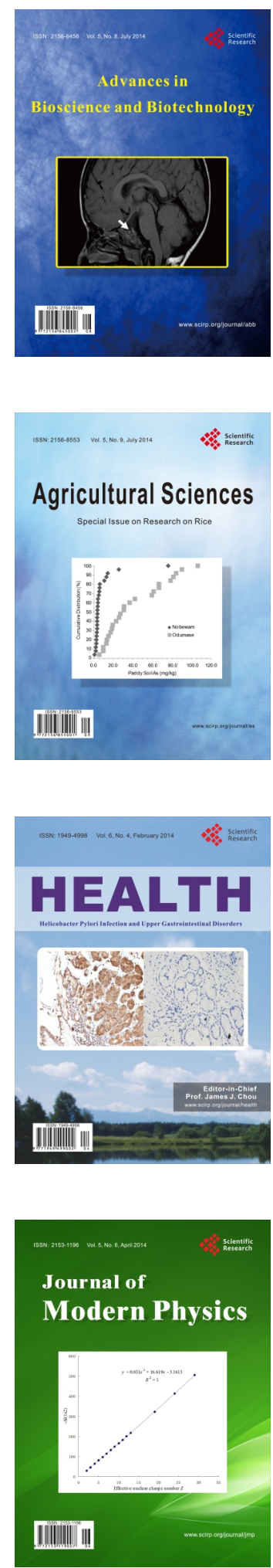
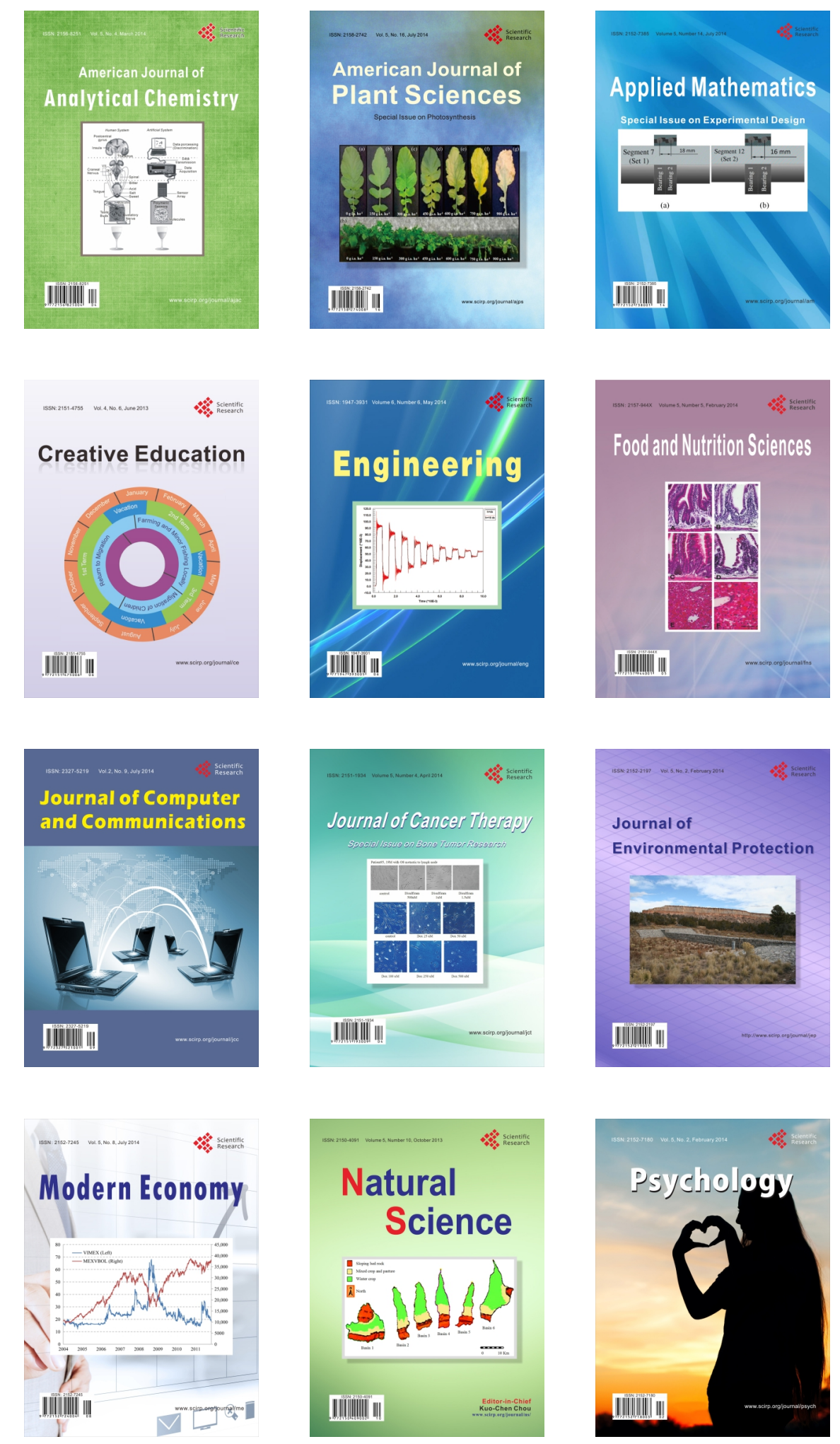